\title{
Adaptabilidade e estabilidade de acessos de uma coleção nuclear de arroz
}

\author{
Luíce Gomes Bueno(1), Rosana Pereira Vianello(1), Paulo Hideo Nakano Rangel(1), Marley Marico Utumi(2), \\ Antônio Carlos Centeno Cordeiro( ${ }^{(3)}$, José Almeida Pereira(4), Daniel Fernandez Franco ${ }^{(5)}$, Francisco Moura Neto ${ }^{(1)}$, \\ João Antônio Mendonça(1), Alexandre Siqueira Guedes Coelho(6), Jaison Pereira de Oliveira(1) e Claudio Brondani(1) \\ (1)Embrapa Arroz e Feijão, Caixa Postal 179, CEP 75375-000 Santo Antônio de Goiás, GO. E-mail: lugobueno@bol.com.br, \\ rosanavb@cnpaf.embrapa.br, phrangel@cnpaf.embrapa.br, brondani@cnpaf.embrapa.br, jaison@cnpaf.embrapa.com, fpmn@cnpaf.embrapa.br, \\ joaoam@cnpaf.embrapa.br (2)Embrapa Rondônia, Caixa Postal 405, CEP 76980-000 Vilhena, RO. E-mail: marleyutumi@hotmail.com \\ (3)Embrapa Roraima, Caixa Postal 133, CEP 69301-970 Boa Vista, RR. E-mail: acarlos@cpafrr.embrapa.br (4)Embrapa Meio-Norte, Caixa

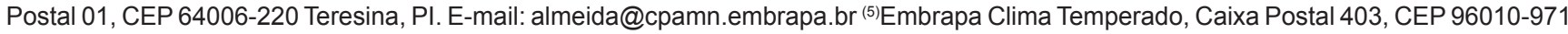 \\ Pelotas, RS. E-mail: daniel.franco@cpact.embrapa.br ${ }^{(6)}$ Universidade Federal de Goiás, Escola de Agronomia e Engenharia de Alimentos, \\ Campus Samambaia, Caixa Postal 131, CEP 74001-970 Goiânia, GO. E-mail: asgcoelho@gmail.com
}

Resumo - O objetivo deste trabalho foi determinar o potencial produtivo e a interação entre genótipos e ambientes em 550 acessos da Coleção Nuclear de Arroz da Embrapa. O desempenho, a adaptação e a estabilidade produtiva de cada genótipo foram avaliados por meio da metodologia de análise dos efeitos principais aditivos e interações multiplicativas (AMMI), em nove experimentos de campo, realizados em seis Estados, em condição de sequeiro e irrigada, durante três anos agrícolas. Foi realizada a análise por meio de modelos lineares mistos, e as estimativas de componentes de variância foram obtidas pelo método de máxima verossimilhança residual (REML), com aplicação do procedimento de melhor predição linear não viesada (BLUP) para a predição dos valores genéticos dos efeitos aleatórios (EBLUP) associados a cada um dos acessos. O grupo de acessos com melhor desempenho foi o de linhagens e cultivares brasileiras (LCB), conforme o esperado. No entanto, foram identificadas variedades tradicionais (VT) entre os genótipos mais produtivos, o que mostra o potencial deste grupo de germoplasma em contribuir com novas fontes de variabilidade genética para programas de melhoramento. Foram identificados acessos superiores quanto à estabilidade, adaptabilidade e produtividade de grãos, entre os quais destacam-se CA880078, CA840182 e CNA00091.

Termos para indexação: Oryza sativa, AMMI, caracterização agronômica, modelo linear misto, recursos genéticos, variedades tradicionais.

\section{Adaptability and stability of accessions of a rice core collection}

\begin{abstract}
The objective of this work was to determine the productive potential and the genotype by environment interaction of 550 accessions of the Embrapa's Rice Core Collection. The performance, and yield adaptability and stability of each genotype was evaluated using the additive main effects and multiplicative interactions (AMMI) approach, in nine field experiments, carried out in six Brazilian states, under dry and irrigated conditions, during three years. The linear mixed model analysis was performed, and the estimates of the variance components were obtained by the residual maximum likelihood (REML), using the best linear unbiased prediction (BLUP) procedure for the prediction of the genotypic values of random effects (EBLUP) associated with each accession. The group of accessions with better performance was the one of lines and cultivars from Brazil (LCB), as expected. However, traditional varieties (VT) were identified among the best yielding genotypes, which shows the potential of this germplasm group to contribute as a new source of genetic variability to breeding programs. Superior accessions were identified for their stability, adaptability and grain yield, among which CA880078, CA840182 and CNA00091stand out.
\end{abstract}

Index terms: Oryza sativa, AMMI, agronomic characterization, mixed linear model, genetic resources, traditional varieties.

\section{Introdução}

Nos programas de melhoramento de arroz, apenas um grupo restrito de genótipos geneticamente semelhantes vem sendo utilizado exaustivamente como genitor, apesar da extensa variabilidade genética disponível nos bancos de germoplasma (Brondani et al., 2006). O principal fator que limita a utilização mais ampla desses acessos é a dificuldade na obtenção e integração de dados, para auxiliar os melhoristas na

Pesq. agropec. bras., Brasília, v.47, n.2, p.216-226, fev. 2012 
escolha de genótipos que efetivamente contribuam para o programa de melhoramento de arroz (Borba et al., 2009).

A caracterização detalhada de todos os acessos de um banco de germoplasma é muitas vezes inviável, fazendo-se necessária a utilização de uma amostragem representativa da variabilidade genética de uma coleção completa, por meio de coleções nucleares. No Brasil, o trabalho pioneiro de desenvolvimento de coleção nuclear de arroz coube a Abadie et al. (2005), que estabeleceu a Coleção Nuclear de Arroz da Embrapa (CNAE) com 550 acessos, estruturada a partir dos dez mil acessos do Banco Ativo de Germoplasma (BAG) da Embrapa Arroz e Feijão.

A caracterização da diversidade dos recursos genéticos do arroz tem sido feita morfologicamentevia descrição de caracteres morfológicos-, agronomicamente e por meio de técnicas de marcadores bioquímicos e moleculares, para avaliar fontes de variabilidade genética e disponibilizá-las a programas de melhoramento (Guarris et al., 2005; Fonseca et al., 2008; Borba et al., 2009). Para obter o conhecimento detalhado da variabilidade genética presente na CNAE, foi realizada a caracterização por marcadores moleculares (Borba et al., 2009) e proteína de reserva (Silveira et al., 2010). Contudo, a análise por marcadores moleculares não substitui a avaliação agronômica em programas de melhoramento de arroz, já que a planta apresenta diferenças fenotípicas marcantes quando cultivada em ambientes diferenciados (Brondani et al., 2006).

A resposta diferenciada dos genótipos, nos vários ambientes, é um fenômeno natural decorrente da interação de genótipos com ambientes ( $\left.\begin{array}{lll}G & \mathrm{x} & \mathrm{E}\end{array}\right)$ (Eberhart \& Russel, 1966), e seus efeitos permitem a identificação de genótipos aptos a um ambiente específico (adaptabilidade), ou de comportamento geral, aptos a vários ambientes (estabilidade).

Este trabalho teve como objetivo determinar o potencial produtivo e a interação de genótipos com ambientes em 550 acessos da Coleção Nuclear de Arroz da Embrapa.

\section{Material e Métodos}

Foram avaliados 550 acessos da Coleção Nuclear de Arroz da Embrapa (CNAE) (Abadie et al., 2005), que é estruturada em subgrupos de acordo com a origem do material e do sistema de cultivo (Tabela 1). A lista completa dos acessos avaliados e maiores informações podem ser encontradas em http://www.cnpaf.embrapa. br/cnae.

Os experimentos para caracterização da produtividade dos acessos da CNAE foram localizados em Goiás, Roraima, Rio Grande do Sul, Mato Grosso, Piauí e Rondônia. Foram instalados nove ensaios, divididos em dois sistemas de cultivo (irrigado e sequeiro) e em três anos agrícolas (2004/2005, 2005/2006 e 2006/2007, denominados 2004, 2005 e 2006, respectivamente) (Tabela 2). Os experimentos foram instalados em delineamento de blocos aumentados de Federer (Federer, 1956), com 23 blocos, e foram adicionadas quatro testemunhas (BR IRGA 409, BRS Caiapó, BRS Colosso e Metica 1). As parcelas experimentais constituíram-se de três fileiras de $4 \mathrm{~m}$, espaçadas em $0,30 \mathrm{~m}$ entre linhas, que perfaziam uma área útil de 3,6 $\mathrm{m}^{2}$, com densidade de plantio de 100 sementes por metro. A produtividade (Prod) foi estimada pela pesagem dos grãos colhidos na área útil da parcela, convertida em quilogramas por hectare.

Foram realizadas, inicialmente, as análises de variância individual da produtividade de cada experimento. Assumiu-se a abordagem metodológica de modelos lineares mistos, em razão da existência de dois tipos de tratamento (testemunhas e acessos) com efeitos de natureza diferente, fixos para testemunhas e aleatórios para acessos, tendo-se considerado um modelo estatístico baseado na proposta de Scott \& Milliken(1993), cujo detalhamento pode ser encontrado em Duarte (2000).

Tabela 1. Número de acessos dos estratos da Coleção Nuclear de Arroz da Embrapa avaliados nos diferentes sistemas de cultivo.

\begin{tabular}{lcccc}
\hline Estratos $^{(1)}$ & \multicolumn{3}{c}{ Sistema de cultivo } & Total \\
\cline { 2 - 4 } & \multicolumn{3}{c}{ Sequeiro Irrigado Facultativo } & \\
\hline Linhagens e cultivares brasileiras & 57 & 37 & - & 94 \\
Linhagens e cultivares introduzidas & 76 & 72 & - & 148 \\
Variedades tradicionais & 148 & 77 & 83 & 308 \\
\hline Total & 281 & 186 & 83 & 550 \\
\hline
\end{tabular}

${ }^{(1)}$ Linhagens e cultivares brasileiras, genótipos melhorados por programas de melhoramento da Embrapa e outras instituições brasileiras; linhagens e cultivares introduzidas, genótipos provenientes de programas de melhoramento de outros países; variedades tradicionais, acessos obtidos por expedições de coleta de germoplasma realizadas em vários estados do Brasil, durante o período de 1979 a 2002. ${ }^{(2)}$ Linhagens adaptadas aos sistemas de cultivo sequeiro e irrigado. 
Os ensaios foram agrupados para as análises univariadas conjuntas, conforme sistema de cultivo e análise geral entre todos os experimentos. No procedimento de análise, foi considerada a recuperação das informações interblocos e intergenotípica. Para tanto, foram considerados efeitos aleatórios para blocos e genótipos (exceto testemunhas), além do erro experimental. As estimativas de componentes de variância foram obtidas pelo método máxima verossimilhança residual (REML), descrito por Patterson \& Thompson (1971), e a predição dos valores genéticos de cada indivíduo foi realizada com o procedimento de melhor predição linear não viesada (BLUP). Os valores preditos dos efeitos aleatórios (EBLUP), associados a cada um dos acessos, contêm uma parte atribuída à estimativa $\hat{\mu}_{\mathrm{p}}$ constante, e outra parte referente ao efeito genotípico $\left(\widetilde{\mathrm{g}}_{\mathrm{i}}\right)$ particular de cada acesso. A utilização de modelos lineares mistos, em estudos de estabilidade e adaptabilidade, apresenta resultados superiores à abordagem convencional de análise, baseada no desempenho médio dos genótipos, principalmente quando ocorrem dados desbalanceados (Balestre et al., 2010; Borges et al., 2010).

Por meio da análise de efeitos principais aditivos e interações multiplicativas (AMMI), fez-se um estudo detalhado do comportamento dos genótipos em relação aos ambientes avaliados, o que permitiu mensurar a adaptabilidade e a estabilidade produtiva de cada genótipo. O modelo da análise pode ser expresso por,

$$
\mathrm{Y}_{\mathrm{ij}}=\mu+\mathrm{g}_{\mathrm{i}}+\mathrm{a}_{\mathrm{j}}+\sum_{\mathrm{k}=1}^{\mathrm{n}} \lambda_{\mathrm{k}} \gamma_{\mathrm{ik}} \alpha_{\mathrm{jk}}+\rho_{\mathrm{ij}}+\overline{\mathrm{e}}_{(\mathrm{i}) \mathrm{j}}
$$

em que: $Y_{i j}$ é a resposta média do genótipo i ( $i=1$, $2, \ldots, G$ genótipos), no ambiente $\mathrm{j}(\mathrm{j}=1,2, \ldots, \mathrm{A}$ ambientes); $\mu$ é a constante comum (média geral); $\mathrm{g}_{\mathrm{i}}$ é o efeito aleatório do genótipo i $(i=1,2, \ldots, g)$; $a_{j}$ é o efeito fixo do ambiente $\mathrm{j}(\mathrm{j}=1,2, \ldots, \mathrm{a}) ; \lambda_{\mathrm{k}}$ é o k-ésimo valor singular (escalar) da matriz de interações original (denotada por GA); $\gamma_{\mathrm{ik}}$ é o elemento correspondente ao genótipo i, no vetor singular k (coluna da matriz GA); $\alpha_{\mathrm{jk}}$ é o elemento correspondente ao ambiente $\mathrm{j}$, no vetor singular k (linha da matriz GA); $\rho_{\mathrm{ij}}$ é o ruído associado ao termo $(\mathrm{ga})_{\mathrm{ij}}$ da interação clássica do genótipo i com $\mathrm{o}$ ambiente $\mathrm{j} ; \overline{\mathrm{e}}_{(\mathrm{il)j}}$ é o erro experimental médio.

\section{Resultados e Discussão}

Foi observada grande amplitude de variação quanto à produtividade, entre os 550 acessos da CNAE. Os valores genotípicos $(\hat{\mu}+\hat{g} i+\hat{g} e)$ associados a cada um dos acessos, preditos em cada ambiente, também apresentaram considerável variação quanto à produtividade, verificada pela comparação entre os valores médios, máximos e mínimos em cada experimento (Tabela 3). A amplitude dos dados foi menor entre os experimentos de sequeiro, com médias dos acessos de $270 \mathrm{~kg} \mathrm{ha}^{-1}$ (Santo Antônio de GoiásSeq2004) até mais de $5.646 \mathrm{~kg} \mathrm{ha}^{-1}$ (Teresina-Seq2006). $\mathrm{O}$ arroz cultivado no sistema de sequeiro apresenta, em geral, baixa produtividade, além de qualidade inferior em comparação aos grãos oriundos de cultura irrigada. Os frequentes períodos de deficiência hídrica a que a cultura está sujeita durante o ciclo dificulta o enchimento contínuo dos grãos e que as plantas atinjam seu potencial produtivo (Crusciol et al., 2003). Entre os experimentos irrigados, foram identificados valores de produtividade entre $2.756 \mathrm{~kg} \mathrm{ha}^{-1}$ (Goianira-Irr2004) e $9.179 \mathrm{~kg} \mathrm{ha}^{-1}$ (Pelotas-Irr2005) (Tabela 3).

Tabela 2. Localidades, ano agrícola, coordenadas geográficas e altitudes dos experimentos realizados para a caracterização da Coleção Nuclear de Arroz da Embrapa.

\begin{tabular}{|c|c|c|c|c|c|c|}
\hline \multirow[t]{2}{*}{ Experimento } & \multirow[t]{2}{*}{ Localidade e ano } & \multirow{2}{*}{$\begin{array}{l}\text { Sistema de } \\
\text { cultivo }\end{array}$} & \multicolumn{3}{|c|}{ Coordenadas } & \multirow[t]{2}{*}{ Altitude (m) } \\
\hline & & & $\mathrm{S}$ & $\mathrm{N}$ & W & \\
\hline$\overline{1}$ & Santo Antônio de Goiás, GO (2004/2005) & Sequeiro & $16^{\circ} 28^{\prime}$ & - & $49^{\circ} 17^{\prime}$ & 779 \\
\hline 2 & Sinop, MT (2005/2006) & Sequeiro & $11^{\circ} 51^{\prime}$ & - & $55^{\circ} 30^{\prime}$ & 345 \\
\hline 3 & Teresina, PI (2006/2007) & Sequeiro & $5^{\circ} 05^{\prime}$ & - & $42^{\circ} 48^{\prime}$ & 72 \\
\hline 4 & Vilhena, RO (2006/2007) & Sequeiro & $12^{\circ} 47^{\prime}$ & - & $60^{\circ} 05^{\prime}$ & 600 \\
\hline 5 & Goianira, GO (2004/2005) & Irrigado & $16^{\circ} 26^{\prime}$ & - & $49^{\circ} 23^{\prime}$ & 728 \\
\hline 6 & Goianira, GO (2005/2006) & Irrigado & $16^{\circ} 26^{\prime}$ & - & $49^{\circ} 23^{\prime}$ & 728 \\
\hline 7 & Boa Vista, RR (2004/2005) & Irrigado & - & $2^{\circ} 48^{\prime}$ & $60^{\circ} 39^{\prime}$ & 61 \\
\hline 8 & Uruguaiana, RS (2004/2005) & Irrigado & $29^{\circ} 45^{\prime}$ & - & $57^{\circ} 05^{\prime}$ & 74 \\
\hline 9 & Pelotas, RS (2005/2006) & Irrigado & $31^{\circ} 52^{\prime}$ & - & $52^{\circ} 21^{\prime}$ & 13 \\
\hline
\end{tabular}


A utilização dos valores genéticos preditos (EBLUP), associados a cada um dos acessos, permitiu a ordenação e a comparação mais eficiente, pois o processo de predição leva em conta a conexão existente entre informações dos blocos e experimentos e promove um ajuste das médias observadas que é mais coerente e representativo da realidade (Resende et al., 1996). Além disso, os valores preditos corresponderam aos valores observados livres do efeito do ambiente. Neste sentido, a apresentação dos resultados foi feita com base nessas predições dos valores genéticos e não nos valores observados em campo.

A média geral de produtividade dos experimentos variou de $1.470 \mathrm{~kg} \mathrm{ha-1}$ (Vilhena-Seq2006) a $6.536 \mathrm{~kg} \mathrm{ha}^{-1}$ (Pelotas-Irr2005), o que mostra uma tendência de ampla variação ambiental da expressão do caráter, nos locais onde os genótipos foram avaliados.

Entre os acessos de maior produtividade, considerando-se como referência o valor médio de todos os acessos mais dois desvios-padrão, estiveram presentes acessos das linhagens e cultivares brasileiras (LCB), linhagens e cultivares introduzidas (LCI) e variedades tradicionais (VT), assim como acessos representativos dos três sistemas de cultivo do arroz no Brasil (irrigado, sequeiro e facultativo) (Tabela 4).

Os acessos do estrato VT - CA940008 (sequeiro), CA960041 (irrigado) e CA960030 (irrigado) apresentaram elevadas médias de produtividade, além de figurarem entre os mais produtivos em grande parte dos ambientes avaliados, distribuídos nas regiões Nordeste, Sul, Centro-Oeste e Norte (Tabela 4). Esse fato reforça a potencialidade adaptativa das VT às diferentes condições de cultivo e de ambiente.

Acessos do grupo VT são constituídos por materiais antigos, provenientes de introduções de outros países ou originadas no próprio país, porém resultantes de um processo contínuo de seleção e adaptação, normalmente realizada por pequenos produtores (Pereira, 2002). Por esse motivo, as variedades tradicionais apresentam variabilidade genética expressiva e são capazes de produzir mesmo sob algum efeito limitante de produtividade como, por exemplo, incidência de doenças e deficit hídrico. Apesar de as variedades tradicionais não apresentarem elevado potencial produtivo, em comparação aos materiais melhorados, elas têm alta estabilidade e adaptação a ambientes com condições mais limitantes à produção, por serem geralmente constituídas por uma combinação de genótipos (Brondani et al., 2006).

A relação $\phi_{\mathrm{b}}$, na maior parte dos ensaios, apresentou estimativas superiores a 0,24 , sendo que apenas em Pelotas Irr2005 observou-se estimativa próxima de zero (Tabela 5). Grandes estimativas $\hat{\sigma}_{\mathrm{e}}^{2}$ podem ser a causa de baixos índices da relação $\phi_{\mathrm{b}} \mathrm{e}$, em associação com baixas estimativas de $\hat{\sigma}_{\mathrm{b}}^{2}$, irão indicar uma pequena diferenciação entre blocos, com heterogeneidade dentro deles, o que muitas vezes pode decorrer de uma escolha de blocos baseada apenas numa medida preventiva, ao invés de ser uma estratificação criteriosa das unidades experimentais (Duarte, 2000).

Elevados índices de $\phi_{\mathrm{b}}$ podem estar associados à falta de homogeneidade das áreas experimentais e a altos valores de $\hat{\sigma}_{\mathrm{b}}^{2}$. No entanto, para este conjunto de experimentos, já eram esperados elevados índices para estas estimativas, principalmente para as variáveis com correlação significativa com florescimento de plantas, uma vez que a distribuição dos blocos foi feita com base nos ciclos reprodutivos dos genótipos, para minimizar os efeitos competitivos entre materiais de diferentes ciclos e portes de planta. Para estas condições, Duarte (2000) enfatiza a vantagem de se utilizar métodos de

Tabela 3. Produtividade de genótipos de arroz em nove experimentos, estimativas dos valores preditos dos efeitos aleatórios e de valores médios, máximos e mínimos, e número de genótipos avaliados.

\begin{tabular}{|c|c|c|c|c|c|c|c|c|c|}
\hline Descrição & $\begin{array}{c}\text { Sto. Ant. Goiás } \\
\text { Seq2004 } \\
\end{array}$ & $\begin{array}{c}\text { Sinop } \\
\text { Seq2005 }\end{array}$ & $\begin{array}{l}\text { Teresina } \\
\text { Seq2006 } \\
\end{array}$ & $\begin{array}{l}\text { Vilhena } \\
\text { Seq2006 }\end{array}$ & $\begin{array}{c}\text { Goianira } \\
\text { Irr2004 }\end{array}$ & $\begin{array}{l}\text { Goianira } \\
\text { Irr2005 }\end{array}$ & $\begin{array}{c}\text { Boa Vista } \\
\text { Irr2004 }\end{array}$ & $\begin{array}{c}\text { Uruguaiana } \\
\text { Irr2004 }\end{array}$ & $\begin{array}{l}\text { Pelotas } \\
\text { Irr2005 } \\
\end{array}$ \\
\hline Número de observações & 510 & 447 & 545 & 537 & 546 & 546 & 530 & 424 & 436 \\
\hline Média geral & $1.772,15$ & $2.635,66$ & $3.764,90$ & $1.469,58$ & $4.641,96$ & $4.697,90$ & $6.014,32$ & $4.917,26$ & $6.536,10$ \\
\hline Média de acessos & $1.383,30$ & $2.290,90$ & $3.203,80$ & $1.108,41$ & $4.117,80$ & $4.258,80$ & $5.080,70$ & $4.032,70$ & $6.272,40$ \\
\hline Média de testemunhas & $1.869,36$ & $2.721,85$ & $3.905,18$ & $1.559,88$ & $4.773,00$ & $4.807,68$ & $6.247,73$ & $5.138,40$ & $6.602,03$ \\
\hline Máximo & $3.030,09$ & $2.525,26$ & $5.645,91$ & $1.794,89$ & $5.777,18$ & $5.261,16$ & $7.664,48$ & $4.220,78$ & $9.178,86$ \\
\hline Mínimo & 270,04 & $2.081,35$ & $2.057,73$ & 750,50 & $2.756,06$ & $3.114,30$ & $3.307,83$ & $3.902,84$ & $4.438,51$ \\
\hline $\mathrm{CV}(\%)$ & 22,08 & 33,64 & 17,78 & 26,85 & 26,73 & 23,23 & 25,56 & 29,84 & 17,74 \\
\hline
\end{tabular}

${ }^{(1)}$ Ano de realização dos experimentos e sistemas de cultivo: Seq, arroz de sequeiro; Irr, arroz irrigado. 
análises que permitam a recuperação da informação interblocos (contida no parâmetro $\hat{\sigma}_{b}^{2}$ ) geralmente responsáveis pela melhoria da qualidade de estimativas do modelo utilizado.
$\mathrm{Na}$ maioria dos locais avaliados, a maior parte da variação total foi atribuída à variável $\hat{\sigma}_{\mathrm{e}}^{2}$. Alguns fatores contribuíram para a elevação dessas estimativas, como o grande número de genótipos avaliados e a utilização

Tabela 4. Valores genotípicos preditos dos acessos de arroz da Coleção Nuclear de Arroz da Embrapa com melhor produtividade na média dos ambientes, e valor médio das testemunhas, quanto à produtividade de grãos $(\mathrm{kg}$ ha-1 $)$, em nove ambientes e na média dos ambientes ${ }^{(1)}$.

\begin{tabular}{|c|c|c|c|c|c|c|c|c|c|c|c|c|}
\hline $\begin{array}{l}\begin{array}{l}\text { Sistema } \\
\text { cultivo }^{(2)}\end{array} \\
\end{array}$ & $\begin{array}{l}\text { Código } \\
\text { CNAE }^{(3)} \\
\end{array}$ & essos & $\begin{array}{l}\text { Sto. Ant. Goiás } \\
\text { Seq2004(4) }\end{array}$ & $\begin{array}{c}\text { Sinop } \\
\text { Seq2005 }\end{array}$ & $\begin{array}{l}\text { Teresina } \\
\text { Seq2006 } \\
\end{array}$ & $\begin{array}{c}\text { Vilhena } \\
\text { Seq2006 } \\
\end{array}$ & $\begin{array}{c}\text { Goianira } \\
\text { Irr2004 } \\
\end{array}$ & $\begin{array}{l}\text { Goianira } \\
\text { Irr2005 } \\
\end{array}$ & $\begin{array}{l}\text { Boa Vista } \\
\text { Irr2004 }\end{array}$ & $\begin{array}{l}\text { Uruguaiana } \\
\text { Irr2004 }\end{array}$ & $\begin{array}{l}\text { Pelotas } \\
\text { Irr2005 }\end{array}$ & Média \\
\hline $\bar{I}$ & LCI65 & CNA0004566 & $2.108,6$ & - & 061,2 & $1.044,3$ & $\underline{5.137,9}$ & $4.407,9$ & $7.664,5$ & $\underline{4.174,0}$ & $7.385,3$ & $4.497,96$ \\
\hline $\mathrm{S}$ & VT274 & CA92 & 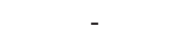 & - & $2.516,5$ & - & $3.917,8$ & $4.134,3$ & 502,8 & 165,1 & $6.662,1$ & $4.483,11$ \\
\hline I & VT289 & CA960041 & 831 & 2.354, & $\underline{5.077 .7}$ & 092,5 & $4.905,3$ & $4.783,4$ & $6.172,6$ & - & $\underline{9.178,9}$ & $4.424,55$ \\
\hline I & LCI20 & CNA0001423 & - & - & $3.104,4$ & - & $3.988,7$ & $4.064,9$ & $5.890,4$ & - & - & $4.262,12$ \\
\hline I & LCI5 & CICA 9 & 812,6 & - & $3.798,4$ & $1.077,1$ & $4.706,6$ & $4.548,4$ & $6.274,6$ & 112,5 & $7.363,9$ & $4.211,76$ \\
\hline $\mathrm{S}$ & LCB56 & CNA0006422 & 62,7 & 2.351 & $3.968,6$ & $\underline{1.624,8}$ & $4.603,8$ & $4.681,8$ & $6.508,8$ & - & $\underline{7.775,6}$ & $4.197,14$ \\
\hline I & VT287 & CA960030 & 040,1 & - & $3.547,3$ & $1.041,5$ & $4.120,9$ & $\underline{5.048,9}$ & $\underline{7.559,6}$ & - & $6.895,7$ & $4.179,14$ \\
\hline 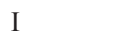 & LCI21 & CNA0001467 & 383,9 & - & $3.815,6$ & $1.262,1$ & $4.612,0$ & $4.424,6$ & $6.527,5$ & $4.004,3$ & $7.363,6$ & $4.174,22$ \\
\hline $\mathrm{F}$ & VT23 & $\mathrm{CA} 7$ & . & $2.240,1$ & $\underline{4.178,7}$ & 932,6 & $4.216,0$ & $4.254,6$ & 1,4 & $\underline{4.183,3}$ & $7.068,7$ & $4.160,69$ \\
\hline I & LCB4 & BRS B & 670,7 & $2.243,9$ & $\underline{4.410,8}$ & $1.071,5$ & $4.973,2$ & $\underline{5.095,5}$ & $\underline{7.122,0}$ & $4.112,5$ & $6.539,5$ & $4.137,75$ \\
\hline I & LCI89 & CNA00 & - & - & $2.947,1$ & - & - & $3.540,0$ & $4.909,2$ & $4.024,8$ & $5.259,6$ & $4.136,16$ \\
\hline $\mathrm{S}$ & LCB29 & CNA00 & 594,7 & $2.295,6$ & $\underline{4.512,9}$ & $1.185,9$ & $4.981,5$ & $4.299,0$ & $7.120,4$ & $4.049,7$ & $7.052,8$ & $4.121,40$ \\
\hline I & VT283 & CA9 & 3,6 & $2.268,0$ & $\underline{4.454,7}$ & $1.132,5$ & $4.097,7$ & $4.570,4$ & $7.020,4$ & - & $\underline{7.648,8}$ & $4.114,51$ \\
\hline I & LCB7 & BRS & & - & $3.841,2$ & $1.100,8$ & $4.534,4$ & & $\underline{6.883,1}$ & 123,2 & $6.523,6$ & $4.114,35$ \\
\hline $\mathrm{S}$ & LCI132 & CNAO & 3,8 & $2.482,5$ & $\underline{4.671,5}$ & $1.160,8$ & $4.759,4$ & $4.368,5$ & $6.259,8$ & $4.014,0$ & $7.513,6$ & $4.101,01$ \\
\hline I & LCB94 & SCS E & & - & $3.843,6$ & $1.162,2$ & $4.646,4$ & $4.469,8$ & 4,1 & $4.064,6$ & $\underline{7.720,0}$ & $4.095,03$ \\
\hline S & LCI73 & CNA0 & 57,2 & - & $3.345,0$ & $1.162,2$ & $\underline{5.430,4}$ & $3.174,3$ & $\underline{6.789 .2}$ & $4.070,9$ & $6.807,3$ & $4.079,56$ \\
\hline I & LCB75 & RS6PI & & $2.335,2$ & $\underline{4.405,1}$ & 992,2 & $4.276,1$ & $4.656,0$ & $\underline{7.247 .1}$ & - & $7.587,8$ & $4.076,71$ \\
\hline I & LCB50 & $\mathrm{CNA}$ & & $2.232,2$ & $\underline{4.397,1}$ & $1.001,0$ & $4.674,5$ & & $\underline{7.121,6}$ & 194,7 & & $4.048,62$ \\
\hline S & VT199 & CA8 & 6,2 & $2.302,8$ & $\underline{4.601,4}$ & $1.172,1$ & $\underline{5.617,7}$ & $4.664,8$ & $\underline{7.318,4}$ & - & $5.217,0$ & $4.041,30$ \\
\hline $\mathrm{F}$ & VT187 & $\mathrm{CA}$ & & $2.279,2$ & $4.035,2$ & $\underline{1.405,3}$ & $\underline{5.168,5}$ & $4.644,3$ & $5.310,9$ & 993,3 & 7,3 & $4.000,60$ \\
\hline $\mathrm{F}$ & VT71 & C & & $2.298,5$ & $\underline{4.406,7}$ & $1.028,9$ & $4.910,9$ & 5,6 & $\underline{6.834,9}$ & $\underline{4.128,1}$ & & $3.909,28$ \\
\hline I & VT39 & $\mathrm{CA} 7$ &, 3 & $2.335,5$ & $3.183,1$ & $\underline{1.794,9}$ & $4.274,6$ & $\underline{4.999,2}$ & $5.721,0$ & $4.074,3$ & $6.741,5$ & $3.877,05$ \\
\hline S & VT236 & CA 87 & $\underline{2.356,9}$ & $2.328,3$ & $2.882,0$ & $1.122,2$ & $\underline{5.777,2}$ & $4.659,3$ & & $4.107,3$ & & $3.864,19$ \\
\hline . & LCI140 & IR36 & 794,3 & $2.208,4$ & $3.172,3$ & 995,4 & $\underline{5.237,8}$ & $4.202,4$ & $6.086,0$ & $\underline{4.148,4}$ & $6.858,1$ & $3.855,91$ \\
\hline S & VT12 & $\mathrm{CA} 7$ & 9,3 & $2.327,6$ & 0,9 & $\underline{1.522,4}$ & $4.529,4$ & $\underline{5.046,4}$ & $5.300,7$ & $4.057,2$ & $7.229,7$ & $3.854,84$ \\
\hline I & LCB 88 & IRGA 418 & $1.847,8$ & $2.291,0$ & $4.099,0$ & $1.330,3$ & $4.641,0$ & $\underline{4.835,8}$ & 0,3 & $\underline{4.147,0}$ & 6.3 & $3.854,17$ \\
\hline I & VT273 & CA940007 & 3,3 & . & 8,0 & 750,5 & $4.038,9$ & $4.572,7$ & 3,8 & $\underline{4.220,8}$ & $\underline{8.080,0}$ & $3.837,25$ \\
\hline S & LCB32 & CNA0004120 & $\underline{2.192,0}$ & $2.317,7$ & $2.531,0$ & $\underline{1.423,3}$ & $4.282,3$ & $\underline{4.888,0}$ & $5.871,5$ & $4.063,4$ & $6.900,1$ & $3.829,91$ \\
\hline S & LCB46 & $\mathrm{CNAC}$ & $\underline{2.333,6}$ & $2.410,3$ & $3.367,5$ & $1.147,5$ & $\underline{5.152,2}$ & $\underline{4.833,1}$ & $4.399,5$ & $4.036,8$ & $6.665,8$ & $3.816,26$ \\
\hline 1 & LCI53 & CNA0003591 & & $2.219,8$ & $2.515,9$ & 945,1 & $4.636,4$ & $3.903,0$ & $6.511,5$ & $\underline{4.206,6}$ & $\underline{7.891,8}$ & $3.800,36$ \\
\hline S & LCI39 & CNA0003287 & $1.756,7$ & $2.315,4$ & $2.976,2$ & $\underline{1.439,6}$ & $3.696,3$ & $4.741,7$ & $5.469,6$ & $4.001,9$ & $\underline{7.801,5}$ & $3.799,87$ \\
\hline $\mathrm{S}$ & LCB80 & IAC 165 & $\underline{2.343,3}$ & $\underline{2.509,4}$ & $3.821,0$ & $1.374,8$ & $\underline{5.133,6}$ & $4.470,3$ & $4.044,7$ & & $6.584,1$ & $3.785,15$ \\
\hline 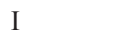 & LCI146 & Oryzica Lhanos 4 & $2.102,1$ & $2.160,9$ & $\underline{4.487,5}$ & $\underline{967,3}$ & $4.750,7$ & $4.689,5$ & $\underline{7.051,2}$ & $4.023,1$ & . & $3.779,01$ \\
\hline $\mathrm{S}$ & LCI74 & CNA0004759 & $1.359,2$ & $2.227,7$ & $2.697,2$ & 922,5 & $\underline{5.282,6}$ & $3.773,0$ & $\underline{6.833,1}$ & $4.088,7$ & 548,8 & $3.748,10$ \\
\hline 1 & VT255 & CA870181 & - & - & $\underline{5.057,5}$ & 982,2 & $\underline{5.143,6}$ & $3.764,7$ & - & - & - & $3.737,03$ \\
\hline I & - & BR IRGA $409^{(5)}$ & $1.849,13$ & $2.655,80$ & $3.567,10$ & $1.697,29$ & $5.988,70$ & $5.442,50$ & $6.641,50$ & $5.270,40$ & $8.285,90$ & $4.599,81$ \\
\hline $\mathrm{S}$ & - & BRS Caiapó & $2.037,83$ & $2.634,00$ & $2.929,30$ & $1.468,70$ & $2.948,90$ & $5.720,20$ & $4.502,90$ & $4.464,20$ & $6.756,80$ & $3.718,09$ \\
\hline S & - & BRS Colosso & $2.121,01$ & $4.057,20$ & $4.310,80$ & $1.534,60$ & $3.911,00$ & $4.785,00$ & $3.761,30$ & $6.111,10$ & $5.623,30$ & $4.023,92$ \\
\hline 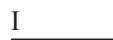 & - & Metica_1 & $1.469,46$ & $1.540,40$ & $4.813,50$ & $1.538,92$ & $6.243,40$ & $3.283,00$ & $10.085,20$ & $4.707,90$ & $5.742,10$ & $4.380,43$ \\
\hline
\end{tabular}

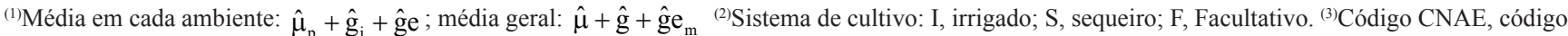
de registro da Coleção Nuclear de Arroz da Embrapa: LCB, linhagens e cultivares brasileiras; LCI, linhagens e cultivares introduzidas; VT, variedades tradicionais. ${ }^{(4)}$ Ano de realização dos experimentos e sistemas de cultivo: Seq, arroz de sequeiro; Irr, arroz irrigado. ${ }^{(5)}$ BR IRGA 409, BRS Caiapó, BRS Colosso, Metica_1, testemunhas. Valores genotípicos sublinhados compreendem acessos que estão entre os vinte mais produtivos em cada ambiente. 
de blocos grandes, com heterogeneidade dentro e pouca diferenciação entre si, o que ocorre com maior facilidade nos experimentos irrigados. Neste sistema de cultivo, há tendência de maior uniformidade do ambiente experimental, em razão da disponibilidade constante de água e da redução na competição com plantas invasoras. Em relação à razão $\phi_{\mathrm{g}}$, observou-se variação nas estimativas entre os diferentes experimentos, de 0,038 a 1,94 (Tabela 5). De modo geral, apesar de não representar a maior parcela da variação fenotípica total, as estimativas do componente $\hat{\sigma}_{\mathrm{g}}^{2}$, observadas nos diferentes locais, fornecem uma confirmação da variabilidade genética presente na coleção de acessos avaliados da CNAE.

$\mathrm{Na}$ análise conjunta, verificou-se que o componente da variância referente aos acessos ( $\hat{\sigma}_{\mathrm{g}}^{2}$ ) foi superior em comparação ao da interação genótipos x ambientes $\left(\hat{\sigma}_{\mathrm{g}_{\mathrm{x}}^{\mathrm{a}}}^{2}\right)$ (Tabela 6). Nessa situação, há ocorrência de maior influência do efeito genotípico na produtividade, o que confirma a existência de variabilidade genética entre os acessos. O componente do efeito de bloco dentro de ambiente $\left(\hat{\sigma}_{\mathrm{b}_{\mathrm{x}}^{\mathrm{a}}}^{2}\right)$ foi responsável pela maior parcela da variação, seguido dos efeitos de genótipo e da interação $\mathrm{G} \times \mathrm{E}$.

$\mathrm{O}$ baixo índice encontrado para o componente de variância ambiental pode ser considerado negligenciável, pois o fator ambiental é de efeito fixo e há nestes resultados um pequeno resíduo, em razão da condição de aninhamento do efeito aleatório de bloco dentro do ambiente. Os grupos de acessos avaliados no presente estudo caracterizam-se pela ampla variabilidade e adaptação (principalmente as variedades tradicionais) e, para um caráter como a produtividade de grãos, que aparentemente mostra grande diferença ambiental (região, altitude, latitude e longitude, temperatura), esperava-se que fossem detectados efeitos de interação acessos x ambientes também significativos. No entanto, alguns aspectos podem estar relacionados a essa ausência de significância, principalmente em consequência das diferentes condições de cultivo envolvidas. Quando se realiza a avaliação, em separado, dos grupos de experimentos irrigados e sequeiros, verifica-se que a análise conjunta dos experimentos irrigados apresenta comportamento semelhante ao da análise realizada com todos os nove locais, e não há significância para os efeitos da interação G x E. Para a análise conjunta dos experimentos de sequeiro, a significância foi detectada tanto para a interação testemunhas $\mathrm{x}$ ambientes, quanto para $\mathrm{G} \times \mathrm{E}$.

O arroz, por ser uma planta tradicionalmente hidrófila, adaptada a áreas encharcadas, tem a produtividade favorecida em condições irrigadas, em que há maior chance de os genótipos expressarem todo o seu potencial produtivo (Vieira et al., 1999). Além disso, nessas condições há maior uniformidade dos ambientes, menor ocorrência de plantas invasoras e, consequentemente, menor competição entre plantas. O sistema de cultivo irrigado, para a cultura do arroz, possibilita que as condições experimentais tornem-se mais uniformes entre diferentes locais, desde que não haja grande variação de temperatura ou fotoperíodo (Peng et al., 2000). Essa padronização ambiental pode ser a causa de não ocorrer interação significativa entre os acessos e os ambientes. Este fato pode favorecer os programas de melhoramento de plantas, no processo de seleção de genótipos, em que uma opção é a de se identificar materiais com boa adaptação e maior estabilidade fenotípica possível para uma gama de ambientes (Nunes et al., 2002).

Pela abordagem da metodologia AMMI, a produtividade foi analisada com o objetivo principal de se conhecer o comportamento dos genótipos em relação à adaptabilidade e estabilidade nos ambientes.

Tabela 5. Estimativas de componentes de variância de genótipo, bloco e erro $\left(\hat{\sigma}_{\mathrm{g}}^{2}, \hat{\sigma}_{\mathrm{b}}^{2}\right.$ e $\left.\hat{\sigma}_{\mathrm{e}}^{2}\right)$, e suas relações com a variância do erro $\left(\phi_{\mathrm{g}}=\hat{\sigma}_{\mathrm{g}}^{2} / \hat{\sigma}_{\mathrm{e}}^{2} \mathrm{e} \phi_{\mathrm{b}}=\hat{\sigma}_{\mathrm{b}}^{2} / \hat{\sigma}_{\mathrm{e}}^{2}\right)$, para a produtividade avaliada em nove experimentos.

\begin{tabular}{lccccccccc}
\hline Parâmetros & $\begin{array}{c}\text { Sto. Ant. Goiás } \\
\text { Seq2004(1) }\end{array}$ & $\begin{array}{c}\text { Sinop } \\
\text { Seq2005 }\end{array}$ & $\begin{array}{c}\text { Teresina } \\
\text { Seq2006 }\end{array}$ & $\begin{array}{c}\text { Vilhena } \\
\text { Seq2006 }\end{array}$ & $\begin{array}{c}\text { Goianira } \\
\text { Irr2004 }\end{array}$ & $\begin{array}{c}\text { Goianira } \\
\text { Irr2005 }\end{array}$ & $\begin{array}{c}\text { Boa Vista } \\
\text { Irr2004 }\end{array}$ & $\begin{array}{c}\text { Uruguaiana } \\
\text { Irr2004 }\end{array}$ & $\begin{array}{c}\text { Pelotas } \\
\text { Irr2005 }\end{array}$ \\
\hline$\hat{\sigma}_{\mathrm{g}}^{2}$ & 296.902 & 67.676 & 535.398 & 75.835 & 820.418 & 476.475 & 1.440 .588 & 81.866 & 1.215 .717 \\
$\hat{\sigma}_{\mathrm{b}}^{2}$ & 58.272 & 509.247 & 235.016 & 65.243 & 378.522 & 292.237 & 921.043 & 263.559 & 114.838 \\
$\hat{\sigma}_{\mathrm{e}}^{2}$ & 153.155 & 786.075 & 448.161 & 155.698 & 1.539 .444 & 1.191 .380 & 2.364 .062 & 2.153 .412 & 1.344 .350 \\
$\phi_{\mathrm{g}}$ & 1,939 & 0,086 & 1,195 & 0,487 & 0,533 & 0,4 & 0,609 & 0,038 \\
$\phi_{\mathrm{b}}$ & 0,38 & 0,648 & 0,524 & 0,419 & 0,246 & 0,245 & 0,39 & 0,122 & 0,0804 \\
\hline
\end{tabular}

${ }^{(1)}$ Ano de realização dos experimentos e sistemas de cultivo: Seq, arroz de sequeiro; Irr, arroz irrigado. 
Nesse processo, os efeitos genotípicos da interação $G$ x E, obtida a partir da análise de variância conjunta, foram utilizados na decomposição de valores singulares (DVS), para que, numa última etapa, as coordenadas para os eixos singulares de interação, gerados pela DVS, fossem utilizados na representação gráfica dos genótipos e ambientes num gráfico biplot.

Os efeitos da interação original puderam ser decompostos em oito componentes principais (CP), dos quais o segundo eixo foi selecionado para a construção dos gráficos biplots. A contribuição de cada componente principal para explicar a soma de quadrados da interação foi baixa, resultado que normalmente tem sido relatado, inclusive quanto a outras culturas, conforme citado, por exemplo, por Rocha et al. (2004), em ensaios conduzidos com soja, e Pereira et al. (2009) com feijoeiro-comum. Os três primeiros eixos, em conjunto, explicaram $49,91 \%$ da variação (Tabela 7), inferior aos 90,1 e 94,8\% obtidos por Samonte et al. (2005) e Morais et al. (2008), também com a cultura do arroz.

Alguns autores consideraram insatisfatórios os resultados obtidos quando os primeiros componentes principais não explicam um percentual superior a $60 \%$ da variação da interação. Contudo, a utilização de modelos com um número maior do que três componentes, do ponto de vista biológico, também é insatisfatória, além de ser de difícil manipulação, em relação aos possíveis benefícios. Gauch \& Zobel

Tabela 6. Resumo das análises conjuntas com estimativas de componentes de variância $\left(\hat{\sigma}_{\mathrm{g}}^{2}, \hat{\sigma}_{\mathrm{a}}^{2}, \hat{\sigma}_{\mathrm{g}_{\mathrm{x}}^{\mathrm{a}}}^{2}\right.$ e $\left.\hat{\sigma}_{\mathrm{b}_{\mathrm{x}}^{\mathrm{a}}}^{2}\right)$ e suas relações com a variância do erro $\left(\phi_{\mathrm{g}}=\hat{\sigma}_{\mathrm{g}}^{2} / \hat{\sigma}_{\mathrm{e}}^{2}, \phi_{\mathrm{a}}=\hat{\sigma}_{\mathrm{a}}^{2} / \hat{\sigma}_{\mathrm{e}}^{2}\right.$, $\left.\left.\phi_{\mathrm{g}_{\mathrm{x}} \mathrm{a}}=\hat{\sigma}_{\mathrm{g}_{\mathrm{x}} \mathrm{a}}^{2} / \hat{\sigma}_{\mathrm{e}}^{2}\right), \phi_{\mathrm{g}_{\mathrm{x}}}=\hat{\sigma}_{\mathrm{g}_{\mathrm{x}} \mathrm{a}}^{2} / \hat{\sigma}_{\mathrm{e}}^{2}, \phi_{\mathrm{b}_{\mathrm{x}} \mathrm{a}}=\hat{\sigma}_{\mathrm{b}(\mathrm{a})}^{2} / \hat{\sigma}_{\mathrm{e}}^{2}\right)$ quanto ao caráter produtividade de grãos $\left(\mathrm{kg} \mathrm{ha}^{-1}\right)$, considerando-se os grupos de experimentos: geral (todos os ensaios), grupo irrigado e grupo de sequeiro.

\begin{tabular}{lccc}
\hline Parâmetros & Geral & Irrigado & Sequeiro \\
\hline$\hat{\sigma}_{\mathrm{g}}^{2}$ & $284.621,5$ & $611.937,9$ & $112.514,4$ \\
$\hat{\sigma}_{\mathrm{a}}^{2}$ & $5.480,2$ & $7.843,9$ & $1.701,2$ \\
$\hat{\sigma}_{\mathrm{g}_{\mathrm{x}}}^{2}$ & $193.193,6$ & $225.290,7$ & $142.409,1$ \\
$\hat{\sigma}_{\mathrm{b}_{\mathrm{x}} \mathrm{a}}$ & $326.914,3$ & $388.759,5$ & $205.365,2$ \\
$\hat{\sigma}_{\mathrm{e}}^{2}$ & $1.195 .343,4$ & $1.710 .857,0$ & $371.560,3$ \\
$\phi_{\mathrm{g}}$ & 0,238 & 0,358 & 0,303 \\
$\phi_{\mathrm{a}}$ & 0,005 & 0,005 & 0,005 \\
$\phi_{\mathrm{g}_{\mathrm{x}}}$ & 0,162 & 0,132 & 0,383 \\
$\phi_{\mathrm{b}_{\mathrm{x}}}$ & 0,273 & 0,227 & 0,553 \\
\hline Média geral & $3.520,1$ & $4.744,4$ & $1.992,69$ \\
\hline
\end{tabular}

(1996) relatam que os primeiros componentes AMMI captam maior percentagem do desempenho real "padrão" adjacente à interação G x E e de importância agronômica. Apesar da seleção de poucos componentes que possam explicar pequena proporção da variação, a informação fornecida pela metodologia AMMI pode ser considerada superior à fornecida por métodos tradicionais para o estudo da interação (Pereira et al., 2009). Assim, a identificação dos acessos mais estáveis, pelo método AMMI, foi realizada com as informações dos dois primeiros componentes. Foram considerados genótipos mais estáveis aqueles que apresentaram os menores valores da amplitude dos escores, os quais apresentam menor contribuição para a interação.

Com relação aos genótipos em estudo, verificou-se, por meio da metodologia AMMI, uma classificação e interpretação simplificadas dos resultados de estabilidade e adaptabilidade, que identificam com eficácia genótipos superiores (Figura 1 e Tabela 8). Assim, foi possível a identificação de combinações mais específicas dos genótipos com os ambientes, associadas ao potencial produtivo, para utilização desses genótipos como possíveis genitores em programas de melhoramento.

Entre os acessos que mais contribuíram para a interação G x E estão os acessos CNA0002672, CA800072 e Basmati 370, pois apresentaram maior amplitude dos valores preditos (EBLUP) das interações genótipos $\mathrm{x}$ ambientes (Tabela 8). $\mathrm{O}$ acesso CNA0002672, assim como a variedade tradicional CA840018, apesar de sua elevada contribuição para a interação, mostraram adaptabilidade específica ao experimento Boa Vista-Irr2004, evidenciado pelos elevados índices preditos para este local, com ganhos de 786 e $729 \mathrm{~kg} \mathrm{ha}^{-1}$, respectivamente.

Tabela 7. Proporção da soma de quadrados da interação genótipo por ambiente $\left(\mathrm{SQ}_{\mathrm{GxE}}\right)$, explicada por eixo relativo à análise de componentes principais $(\mathrm{CP})$.

\begin{tabular}{lccc}
\hline CP/Eixo & Autovalor & Proporção/CP & Acumulado (\%) \\
\hline CP1 & $4.509,082$ & 0,1995 & 19,9521 \\
CP2 & $3.478,229$ & 0,1539 & 35,3428 \\
CP3 & $3.292,824$ & 0,1457 & 49,9132 \\
CP4 & $2.975,843$ & 0,1317 & 63,0809 \\
CP5 & $2.286,224$ & 0,1012 & 73,1972 \\
CP6 & $2.120,445$ & 0,0938 & 82,5799 \\
CP7 & $1.793,763$ & 0,0794 & 90,5170 \\
CP8 & $1.243,662$ & 0,0550 & 96,0201 \\
CP9 & 899,444 & 0,0398 & 100,0000 \\
\hline
\end{tabular}


No entanto, entre os acessos que menos contribuíram para a interação $\mathrm{G}$ x $\mathrm{E}$ (mais estáveis) encontramse CA880078, CA840182 e CA990001, alocados próximos às coordenadas IPCA de índice zero (Figura 1), tendo apresentado baixas interações com os ambientes de estudo (Tabela 8). Esses acessos apresentaram plasticidade aos diferentes ambientes avaliados, além de rendimentos satisfatórios.

Uma constatação importante é que esses acessos, exceto o CNA0009113, e ainda a maioria dos considerados mais estáveis, são pertencentes ao estrato variedades tradicionais (VT) da CNAE. Os acessos CNA0006130, BRS Bigua, CNA0003490 e CNA0004098, pertencentes ao estrato de linhagens e cultivares brasileiras (LCB), embora tenham apresentado as maiores médias gerais, não foram tão estáveis aos diferentes ambientes, pois mostraram elevados índices das estimativas das interações $\mathrm{G}$ x E.

No experimento Vilhena-Seq2006, ocorreram as menores médias preditas para todos os acessos.

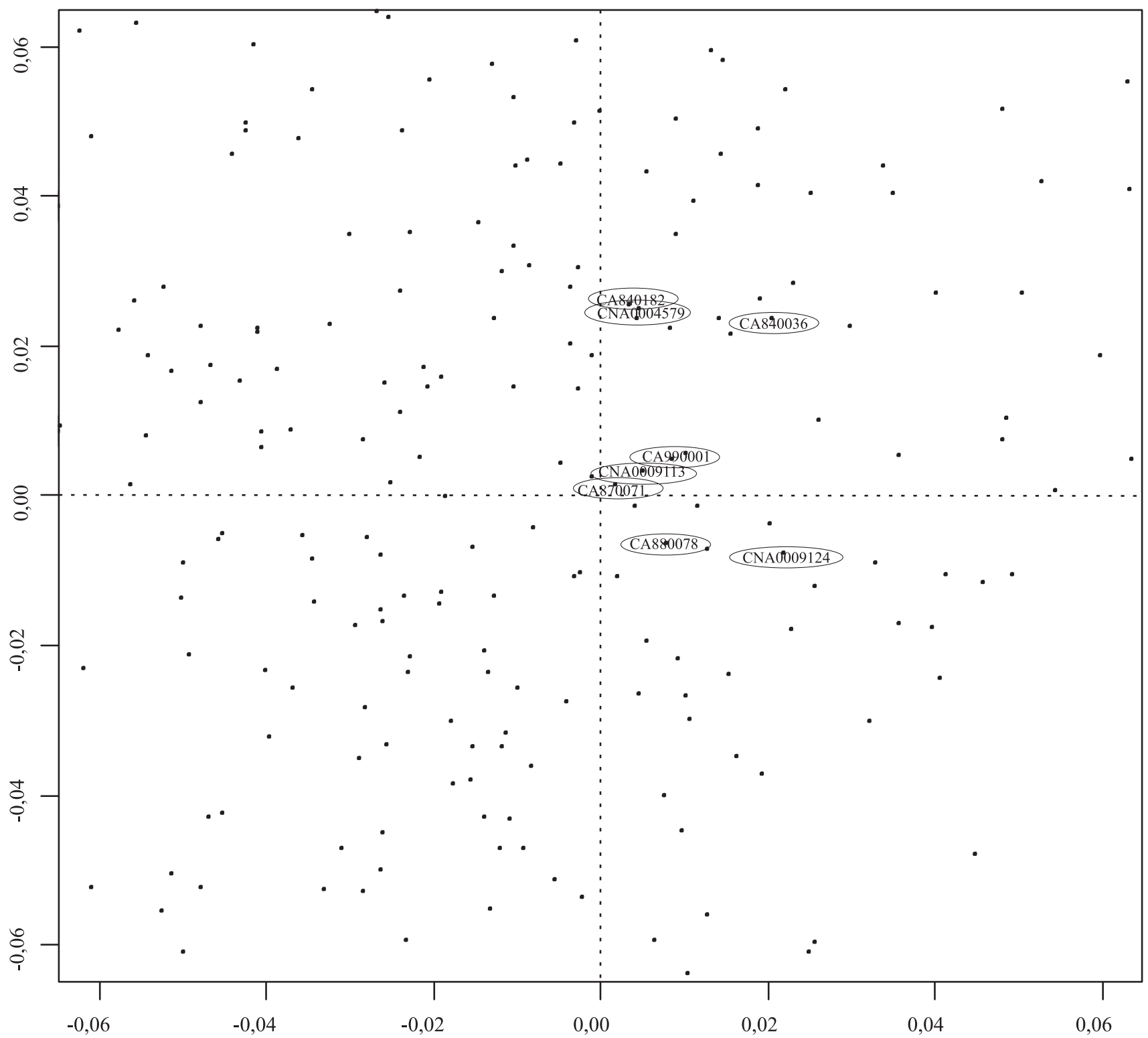

Figura 1. Representação gráfica biplot, ampliada, de acessos da Coleção Nuclear de Arroz da Embrapa, em relação aos dois primeiros componentes principais (CP1 x CP2). Genótipos circulados estão entre os que apresentaram menor amplitude média das estimativas dos valores preditos. 
No entanto, esse foi o ambiente que apresentou menor amplitude de efeitos de interação e mostrou uma estabilidade ambiental típica. Nesta condição, a classificação dos acessos apresentou a menor variação em relação à classificação nos outros experimentos. Segundo Oliveira et al. (2003), a determinação da estabilidade ambiental tem grande importância, pois informa sobre a confiabilidade no ordenamento dos genótipos, em um dado ambiente de teste, em relação à classificação para a média dos ambientes testados. Por esse motivo, esse tipo de ambiente pode ser considerado um local estratégico para a condução de experimentos, em fases preliminares de avaliação de genótipos (Morais et al., 2008). Entre os locais avaliados que mais contribuíram para a interação $\mathrm{G} \times \mathrm{E}$, destacam-se Boa Vista-Irr2004 e Goianira-Irr2004, pelos elevados escores de amplitude de suas estimativas de interação G x E. Quanto aos ambientes, entre os mais estáveis encontra-se Santo Antônio de GoiásSeq2004, mesmo que associado a baixa produtividade dos acessos em relação aos respectivos desempenhos nos demais ensaios.

Tabela 8. Estimativas dos valores preditos dos efeitos aleatórios, das interações genótipos x ambientes, dos 15 genótipos de maior e menor amplitude, em nove experimentos.

\begin{tabular}{|c|c|c|c|c|c|c|c|c|c|c|c|c|}
\hline Acessos & Estrato & $\begin{array}{c}\text { Sistema } \\
\text { de cultivo }\end{array}$ & $\begin{array}{l}\text { Sto. Ant. Goiás } \\
\text { Seq2004 }\end{array}$ & $\begin{array}{c}\text { Sinop } \\
\text { Seq2005 }\end{array}$ & $\begin{array}{l}\text { Teresina } \\
\text { Seq2006 }\end{array}$ & $\begin{array}{l}\text { Vilhena } \\
\text { Seq2006 }\end{array}$ & $\begin{array}{c}\text { Goianira } \\
\text { Irr2004 }\end{array}$ & $\begin{array}{c}\text { Goianira } \\
\text { Irr2005 }\end{array}$ & $\begin{array}{l}\text { Boa Vista } \\
\text { Irr2004 }\end{array}$ & $\begin{array}{l}\text { Uruguaiana } \\
\text { Irr2004 }\end{array}$ & $\begin{array}{l}\text { Pelotas } \\
\text { Irr2005 }\end{array}$ & Amplitude \\
\hline CNA0002672 & LCI & I & 36,5 & 0,4 & $-18,6$ & $-75,7$ & $-19,5$ & $-221,8$ & 785,9 & $-128,9$ & $-283,2$ & $1.069,0$ \\
\hline CA800072 & VT & $\mathrm{F}$ & $-101,7$ & $-40,7$ & $-151,3$ & $-88,9$ & 75,0 & 195,7 & $-385,9$ & $-215,8$ & 660,2 & $1.046,1$ \\
\hline Basmati 370 & LCI & I & 86,8 & 13,6 & $-78,4$ & 40,4 & $-207,4$ & $-438,8$ & $-231,3$ & $-151,7$ & 569,9 & $1.008,6$ \\
\hline IRGA 417 & LCB & I & $-47,8$ & $-63,8$ & 156,9 & 136,0 & $-551,2$ & 275,0 & 66,0 & 430,6 & $-64,4$ & 981,8 \\
\hline CNA0003602 & LCI & I & 71,1 & $-130,0$ & $-279,2$ & $-41,8$ & 118,2 & $-372,7$ & 571,5 & 224,6 & 125,0 & 944,2 \\
\hline CA840018 & VT & $\mathrm{F}$ & $-25,4$ & $-118,0$ & 26,2 & $-45,2$ & $-54,9$ & $-136,5$ & 729,1 & $-209,7$ & 159,1 & 938,8 \\
\hline CA780071 & VT & S & $-49,2$ & 297,7 & $-56,7$ & 74,6 & $-342,8$ & 130,3 & $-589,1$ & $-24,6$ & 342,7 & 931,8 \\
\hline CNA0005342 & LCB & S & 112,6 & $-223,0$ & 21,6 & 13,0 & $-516,5$ & 64,8 & 242,3 & 370,9 & $-181,6$ & 887,4 \\
\hline CNA0006943 & LCI & I & 15,8 & $-202,8$ & $-58,6$ & $-92,1$ & 282,4 & $-96,6$ & 677,8 & $-86,0$ & $-85,9$ & 880,6 \\
\hline IRGA 420 & LCB & I & $-85,5$ & $-139,3$ & 167,5 & $-76,4$ & $-96,2$ & 179,7 & $-265,1$ & 595,4 & $-26,1$ & 860,5 \\
\hline CNA0004759 & LCI & S & $-72,8$ & $-151,3$ & $-179,5$ & $-128,2$ & 396,0 & $-285,7$ & 565,9 & 157,9 & 22,2 & 851,6 \\
\hline CNA0006130 & LCB & I & $-217,6$ & $-241,7$ & 166,8 & $-190,0$ & 78,7 & $-4,4$ & 607,5 & 467,8 & 130,5 & 849,2 \\
\hline BRS Bigua & LCB & I & $-83,9$ & $-250,0$ & 156,5 & $-167,3$ & 180,4 & 251,9 & 596,0 & 164,7 & $-88,0$ & 845,9 \\
\hline CNA0006910 & LCI & I & $-10,8$ & 79,2 & 173,3 & $-122,2$ & 476,6 & 31,1 & $-367,2$ & $-122,1$ & 165,7 & 843,8 \\
\hline CA870179 & VT & $\mathrm{F}$ & $-209,4$ & 139,8 & 91,4 & $-85,7$ & 200,1 & $-146,8$ & 544,0 & 193,4 & $-296,9$ & 840,8 \\
\hline CNA0006187 & $\mathrm{LCB}$ & S & $-45,2$ & 78,7 & $-45,6$ & 41,4 & 66,7 & 88,1 & $-63,7$ & 156,3 & 101,3 & 220,0 \\
\hline CA820071 & VT & S & 54,7 & $-42,4$ & $-53,1$ & 31,4 & $-90,2$ & $-88,1$ & 0,2 & $-163,1$ & 30,0 & 217,7 \\
\hline CNA0001118 & LCB & S & 124,1 & $-71,9$ & $-91,6$ & 16,3 & 94,7 & $-77,1$ & $-38,7$ & $-66,0$ & $-56,4$ & 215,6 \\
\hline CNA0004308 & LCI & I & $-38,2$ & 32,8 & $-74,6$ & 55,5 & $-132,5$ & $-60,9$ & 70,5 & $-54,2$ & $-141,3$ & 211,8 \\
\hline CA810017 & $\mathrm{VT}$ & S & $-69,6$ & 10,8 & $-16,6$ & 73,5 & $-76,7$ & 30,8 & $-34,1$ & $-94,3$ & 98,9 & 193,2 \\
\hline CA840032 & VT & $\mathrm{F}$ & 87,9 & 113,1 & $-66,8$ & 24,3 & $-78,7$ & 83,2 & $-48,6$ & 4,0 & $-24,3$ & 191,8 \\
\hline CNA0001416 & LCB & I & 120,6 & 42,8 & 131,9 & 10,4 & 21,3 & $-11,2$ & 62,5 & $-58,9$ & 98,2 & 190,7 \\
\hline CA870071 & VT & S & 61,0 & $-2,0$ & $-97,0$ & 40,5 & 51,2 & 35,5 & $-2,8$ & 92,7 & 17,2 & 189,6 \\
\hline CNA0009124 & LCI & S & $-103,1$ & $-29,7$ & $-27,8$ & 61,9 & 58,7 & $-17,4$ & 82,9 & $-89,5$ & $-69,0$ & 186,0 \\
\hline CNA0009113 & LCI & S & 114,5 & $-5,2$ & $-31,2$ & $-6,1$ & 8,9 & 137,9 & 65,7 & $-20,1$ & $-41,5$ & 179,3 \\
\hline CNA0009415 & LCI & S & $-12,9$ & 51,0 & 34,2 & 39,8 & 9,6 & $-104,4$ & $-115,1$ & $-100,6$ & 45,4 & 166,0 \\
\hline CA840036 & VT & S & 72,6 & $-52,3$ & $-46,4$ & 13,0 & $-16,5$ & $-0,5$ & 93,5 & 112,9 & 2,9 & 165,1 \\
\hline CA990001 & VT & S & $-8,5$ & $-106,0$ & $-113,3$ & 35,2 & 49,8 & 14,1 & $-0,5$ & $-18,9$ & $-106,3$ & 163,0 \\
\hline CA840182 & VT & S & $-22,6$ & 77,1 & $-72,5$ & $-0,9$ & $-63,3$ & 69,7 & 63,7 & 33,5 & $-61,6$ & 149,5 \\
\hline CA 880078 & VT & $\mathrm{S}$ & 72,8 & $-37,1$ & $-21,1$ & 42,9 & 36,6 & 91,2 & 52,0 & $-7,1$ & 0,5 & 128,3 \\
\hline \multicolumn{3}{|l|}{ Amplitude total $^{(2)}$} & 541,9 & 732,7 & 838,1 & 436,1 & $1.122,0$ & 989,7 & $1.375,0$ & 948,4 & $1.067,2$ & - \\
\hline
\end{tabular}

(1)Ano de realização dos experimentos e sistemas de cultivo: Seq, arroz de sequeiro; Irr, arroz irrigado. ${ }^{(2)}$ Amplitude dos escores de todos os acessos avaliados em cada experimento. 


\section{Conclusões}

1. Há ampla variabilidade genética quanto à produtividade entre os acessos da Coleção Nuclear de Arroz da Embrapa.

2. A maioria dos acessos mais estáveis pertence ao estrato variedades tradicionais (VT) da Coleção Nuclear de Arroz da Embrapa, o que mostra o potencial deste grupo de germoplasma em contribuir com novas fontes de variabilidade genética para programas de melhoramento.

3. Foram identificados acessos superiores quanto à estabilidade, adaptabilidade e produtividade de grãos, com destaque para CA880078, CA840182 e CNA00091.

\section{Agradecimentos}

À Empresa Brasileira de Pesquisa Agropecuária e ao Conselho Nacional de Desenvolvimento Científico e Tecnológico, pelo apoio financeiro.

\section{Referências}

ABADIE, T.; CORDEIRO, C.M.T.; FONSECA, J.R.; ALVES, R. de B. das N.; BURLE, M.L.; BRONDANI, C.; RANGEL, P.H.N.; CASTRO, E. da M.; SILVA, H.T. da; FREIRE, M.S.; ZIMMERMANN, F.J.P.; MAGALHÃES, J.R. Construção de uma coleção nuclear de arroz para o Brasil. Pesquisa Agropecuária Brasileira, v.40, p.129-136, 2005.

BALESTRE, M.; SANTOS, V.B. dos; SOARES, A.A.; REIS, M.S. Stability and adaptability of upland rice genotypes. Crop Breeding and Applied Biotechnology, v.10, p.357-363, 2010.

BORBA, T.C. de O.; BRONDANI, R.P.V.; RANGEL, P.H.N.; BRONDANI, C. Microsatellite marker-mediated analysis of the Embrapa Rice Core Collection genetic diversity. Genetica, v.137, p.293-304, 2009.

BORGES, V.; SOARES, A.A.; REIS, M.S.; RESENDE, M.D.V. de; CORNÉLIO, V.M.O.; LEITE, N.A.; VIEIRA, A.R. Desempenho genotípico de linhagens de arroz de terras altas utilizando metodologia de modelos mistos. Bragantia, v.69, p.833-842, 2010.

BRONDANI, C.; BORBA, T.C.O.; RANGEL, P.H.N.; BRONDANI, R.P.V. Determination of genetic variability of traditional varieties of Brazilian rice using microsatellite markers. Genetics and Molecular Biology, v.29, p.676-684, 2006.

CRUSCIOL, C.A.C.; ARF, O.; SORATTO, R.P.; ANDREOTTI, M.; RODRIGUES, R.A.F. Produtividade e qualidade industrial de grãos de arroz de terras altas em função de lâminas de água no sistema irrigado por aspersão. Acta Scientiarum. Agronomy, v.25, p.125-130, 2003.
DUARTE, J.B. Sobre o emprego e a análise esstatística do delineamento em blocos aumentados no melhoramento genético vegetal. 2000. 293p. Tese (Doutorado) - Escola Superior de Agricultura Luiz de Queiroz, Piracicaba.

EBERHART, S.A.; RUSSEL, W.A. Stability parameters for comparing varieties. Crop Science, v.6, p.36-40, 1966.

FEDERER, W.T. Augmented (or hoonuiaku) designs. Hawaiian Planter's Records, v.55, p.191-208, 1956.

FONSECA, J.R.; NEVES, P. de C.F.; CUTRIM, V. dos A.; OLIVEIRA, J.P.; SILVA, J.B.V. da; GUSMÃO, A.R.E.; FARIA, J.M. Avaliação agronômica de linhagens de arroz irrigado de viveiros internacionais. Santo Antônio de Goiás: Embrapa Arroz e Feijão, 2008. 16p. (Embrapa Arroz e Feijão. Documentos, 223).

GARRIS, A.J.; TAI, T.H.; COBURN, J.; KRESOVICH, S.; MCCOUCH, S.R. Genetic structure and diversity in Oryza sativa L. Genetics, v.169, p.1631-1638, 2005.

GAUCH, H.G.; ZOBEL, R.W. AMMI analysis of yield trials. In: KANG, M.S.; GAUCH, H.G. (Ed.). Genotype by environment interaction. Boca Raton: CRC Press, 1996. p.85-122.

SAMONTE, S.O.P.B.; WILSON, L.T.; MCCLUNG, A.M.; MEDLEY, J.C. Targeting cultivars onto rice growing environments using AMMI and SREG GGE biplot analyses. Crop Science, v.45, p.2414-2424, 2005.

MORAIS, L.K. de; SILVA, R.M. da; CHIORATO, A.F.; AZZINI, L.E.; VILLELA, O.V.; GALLO, P.B.; SAKAI, M.; BASTOS, C.R.; MALAVOLTA, V.M.A. Adaptabilidade e estabilidade fenotípica de genótipos de arroz irrigado para o Estado de São Paulo. Revista Biociências, v.14, p.9-16, 2008.

NUNES, G.H. de S.; REZENDE, G.D.S.P.; RAMALHO, M.A.P.; SANTOS, J.B. dos. Implicações da interação genótipos x ambientes na seleção de clones de eucalipto. Cerne, v.8, p.49-58, 2002.

OLIVEIRA, A.B. de; DUARTE, J.B.; PINHEIRO, J.B. Emprego da análise AMMI na avaliação da estabilidade produtiva em soja. Pesquisa Agropecuária Brasileira, v.38, p.357-364, 2003.

PATTERSON, H.D.; THOMPSON, R. Recovery of inter-block information when block sizes are unequal. Biometrika, v.58, p.545-554, 1971 .

PENG, S.; LAZA, R.C.; VISPERAS, R.M.; SANICO, K.G.; CASSMAN, K.G.; KHUSH, G.S. Grain yield of rice cultivars and lines developed in the Philippines since 1966. Crop Science, v.40, p.307-314, 2000 .

PEREIRA, H.S.; MELO, L.C.; FARIA, L.C. de; DEL PELOSO, M.J.; COSTA, J.G.C. da; RAVA, C.A.; WENDLAND, A. Adaptabilidade e estabilidade de genótipos de feijoeiro-comum com grãos tipo carioca na Região Central do Brasil. Pesquisa Agropecuária Brasileira, v.44, p.29-37, 2009.

PEREIRA, J.A. Cultura do arroz no Brasil: subsídios para a sua história. Teresina: Embrapa Meio-Norte, 2002. 226p.

RESENDE, M.D.V. de; PRATES, D.F.; JESUS, A. de; YAMADA, C.K. Melhor predição linear não viciada (BLUP) de valores genéticos no melhoramento de Pinus. Boletim de Pesquisa Florestal, n.32/33, p.3-22, 1996. 
ROCHA, M.M.; VELLO, N.A.; LOPES, A.C.A.; MAIA, M.C.C. Yield stability of soybean lines using additive main effects and multiplicative interaction analysis - AMMI. Crop Breeding and Applied Biotechnology, v.4, p.391-398, 2004.

SCOTT, R.A.; MILLIKEN, G.A.A. SAS program for analyzing augmented randomized complete-block designs. Crop Science, v.33, p.865-867, 1993.

SILVEIRA, R.D.D.; SANTOS, K.F.d'E.N.; DIDONET, C.C.G.M.; DIDONET, A.D.; BRONDANI, C. Proteínas de reserva de acessos da coleção nuclear de arroz. Pesquisa Agropecuária Brasileira, v.45, p.1441-1447, 2010.

SOUZA, A.P. Biologia molecular aplicada ao melhoramento. In: NASS,L.L.;VALOIS,A.C.C.;MELO,J.S.;VALADARES-INGLIS, M.C. (Ed.). Recursos genéticos e melhoramento - plantas. Rondonópolis: Fundação MT, 2001. p.939-965.

VIEIRA, N.R. de A.; SANTOS, A.B. dos; SANT'ANA, E.P. (Ed.). A cultura do arroz no Brasil. Santo Antônio de Goiás: Embrapa Arroz e Feijão, 1999. 633p.

Recebido em 17 de agosto de 2011 e aprovado em 6 de fevereiro de 2012 